\title{
Multicut is FPT
}

\author{
Nicolas Bousquet \\ Université Montpellier 2 - \\ CNRS LIRMM, 161 rue Ada \\ 34392 Montpellier, France \\ bousquet@lirmm.fr
}

\author{
Jean Daligault \\ Université Montpellier 2 - \\ CNRS LIRMM, 161 rue Ada \\ 34392 Montpellier, France \\ daligault@lirmm.fr
}

\begin{abstract}
Stéphan Thomassé Université Montpellier 2 CNRS LIRMM, 161 rue Ada 34392 Montpellier, France thomasse@lirmm.fr
\end{abstract}

\begin{abstract}
Let $G=(V, E)$ be a graph on $n$ vertices and $R$ be a set of pairs of vertices in $V$ called requests. A multicut is a subset $F$ of $E$ such that every request $x y$ of $R$ is cut by $F$, i.e. every $x y$-path of $G$ intersects $F$. We show that there exists an $O\left(f(k) n^{c}\right)$ algorithm which decides if there exists a multicut of size at most $k$. In other words, the MULTICUT problem parameterized by the solution size $k$ is Fixed-Parameter Tractable.
\end{abstract}

\section{General Terms}

Algorithms

\section{Keywords}

Multicut, FPT, Parameterized Complexity

\section{Categories and Subject Descriptors}

G.2.2 [Mathematics of Computing]: DISCRETE MATHEMATICS - Graph Theory, Graph algorithms

\section{INTRODUCTION}

The study of cuts and flows is one of the most active field in combinatorial optimization. However, if the simplest case involving one source and one sink is algorithmically tractable, the problem becomes hard as soon as one deals with multiple terminals. For instance, given two requests $\left(x_{1}, y_{1}\right)$ and $\left(x_{2}, y_{2}\right)$ in a directed graph $D$, it is NPcomplete to decide if there exist two disjoint directed paths, respectively from $x_{1}$ to $y_{1}$ and from $x_{2}$ to $y_{2}$ [10]. In fact, even deciding if two given vertices belong to a directed circuit is already hard. The picture changes when considering undirected graphs, in which case the celebrated result of Robertson and Seymour [22] asserts that given $k$ requests $\left(x_{1}, y_{1}\right),\left(x_{2}, y_{2}\right), \ldots,\left(x_{k}, y_{k}\right)$, one can decide in cubic time if

\footnotetext{
*Research supported by the ANR Project AGAPE (ANR09-BLAN-0159).
}

Permission to make digital or hard copies of all or part of this work for personal or classroom use is granted without fee provided that copies are not made or distributed for profit or commercial advantage and that copies bear this notice and the full citation on the first page. To copy otherwise, to republish, to post on servers or to redistribute to lists, requires prior specific permission and/or a fee.

STOC'11, June 6-8, 2011, San Jose, California, USA.

Copyright 2011 ACM 978-1-4503-0691-1/11/06 ...\$10.00. there exists $k$ disjoint paths connecting all pairs $x_{i}, y_{i}$. The catch is of course that cubic time refers to the instance size, which we generally denote by $n$. From their work, the complexity of the $k$-path problem is $O\left(f(k) n^{3}\right)$, where $f$ is by no mean polynomial, since the question is NP-complete when $k$ is part of the input.

This result received considerable attention, both since this is the key tool for computing a given minor in a graph, but also because it has opened a breach in the classical NPcomplete $/ \mathrm{P}$ duality. Indeed, the difficulty of the $k$-path problem does not depend on the size of the instance, but rather on the number of paths we are looking for. In other words, the parameter containing the hardness of the problem is the number $k$ of paths. In a more general way, a problem is fixed parameter tractable (FPT) with respect to the parameter $k$ (e.g. solution size, treewidth, ...) if for any instance of size $n$ it can be solved in time $O\left(f(k) n^{c}\right)$ for some fixed $c$. The reader is invited to refer to now classical books by Downey and Fellows [7], Flum and Grohe [8] and Niedermeier [21].

The dual problem of finding disjoint paths from a source $s$ to a sink $t$ is the cut problem where one asks for a set of vertices or edges which deletion separates $s$ from $t$. Menger's theorem, or more generally LP-duality, asserts that the maximum number of disjoint $s t$-paths is equal to the minimum size of a cut. This property no longer holds when considering multiple requests, where the maximum number of disjoint paths connecting requests is only an obvious lower bound for the size of a multicut, i.e. a set of vertices or edges which deletion separates $x_{i}$ from $y_{i}$ for every request $x_{i} y_{i}$. Formally, we have the following problem:

\section{MULTICUT:}

Input: A graph $G$, a set of requests $R$, an integer $k$. Parameter: $k$.

Output: TRUE if there is a multicut of size at most $k$, otherwise FALSE.

The status of this problem is one of the long standing open problems in parameterized complexity. The main result of this paper is to provide an FPT algorithm for MULTICUT. The proofs being slightly less cumbersome in the edge case, we present our work in terms of edge-multicut rather than vertex-multicut. Our last section shows how to translate the algorithm to deal with vertices. Let us now give some formal definitions. Given a graph $G$ and a set $R$ of pairs of distinct vertices called requests, a multicut is a subset $F$ of edges of $G$ such that every $x y$-path, where $x y$ is a request, contains an 
edge of $F$. Equivalently, the two endpoints (or terminals) of every request of $R$ belong to different connected components of $G \backslash F$.

The MULTICUT problem is already hard on trees since VERTEX COVER is equivalent to MULTICUT in stars. Hence MULTICUT is NP-complete and Max-SNP hard. MULTICUT and its variants have raised an extensive literature. These problems play an important role in network issues, such as routing and telecommunication (see [5]).

MULTICUT IN TREES was already a challenging problem. Garg et. al. [11] proved that it admits a factor 2 approximation algorithm. Guo and Niedermeier [15] proved that MULTICUT IN TREES is FPT with respect to the solution size. Bousquet et. al. [1] provided a polynomial kernel.

On general instances, Garg et. al. gave an approximation algorithm for MULTICUT within a logarithmic factor in [9]. However MULTICUT has no constant factor approximation algorithm if Khot's Unique Games Conjecture holds [2]. This fact is a further motivation to the study of the fixed parameterized tractability of MULTICUT. Guo et. al. showed in [14] that MULTICUT is FPT when parameterized by both the treewidth of the graph and the number of requests. Gottlob and Lee in [12] proved a stronger result: MULTICUT is FPT when parameterized by the treewidth of the input structure, namely the input graph whose edge set is completed by the set of request pairs. Recently, Daligault et. al. [6] proved that MULTICUT can be reduced to instances in which the graph $G$ has treewidth bounded in terms of $k$.

Marx proved that MULTICUT is FPT for this latter parameterization [16]. A faster algorithm running in time $O^{\star}(8 \cdot l)^{k}$ was given by Guillemot [13]. Marx et. al. [17] obtained FPT results for more general types of constrained MULTICUT problems through treewidth reduction results. However their treewidth reduction techniques do not yield the tractability of MULTICUT when parameterized only by the solution size. Recently, Marx and Razgon obtained a factor 2 Fixed-Parameter-Approximation for MULTICUT in [18].

Marx and Razgon independently found a proof of the fact that MULTICUT is FPT, with a different approach, see [19].

The outline of our proof is quite pedestrian, even if some of the partial results are still a bit technical. Informally, our goal is to reduce our input graph to a subdivision of a graph with a bounded number of edges. The crucial tool for this reduction is to find a partition of the set of all "reasonable" solutions of the MULTICUT problem into a bounded number of subsets, in which the multicuts are "totally ordered" (this will be formalized in Subsection 5.6). This application of Dilworth's Theorem is maybe the most interesting aspect of our proof. However, it requires an important cleaning of our instance before being applied. It would be interesting to find an adequate partial order on multicuts at an earlier stage of our proof to shorten the argument and get more insight in the structure of multicuts.

In Section 3 we develop some connectivity tools which are used to certify that some requests are irrelevant. In Section 4 , we first compute a vertex-multicut $Y$ of size $k+1$ by iterative compression. We then reduce to the case where each component of $G \backslash Y$ has one or two attachment vertices in $Y$. We show that components with one attachment vertex have only a bounded (in $k$ ) number of terminals. For com- ponents with two attachment vertices, we identify one path (called backbone) where only one edge is chosen in the multicut. In Section 5, we reduce to the case where the backbone is the only path connecting the two attachment vertices. Finally, we show that MULTICUT can be reduced to an instance which is a subdivision of a graph with a bounded number of edges and where the multicut selects a single edge in each of the paths corresponding to the subdivided edges. This case can easily be coded by a 2-SAT instance, which is solvable in polynomial time.

Acknowledgments. This proof was a long process in which several researchers gave us advice or participated in some of the partial results. One of our important tools, Lemma 7, is the crucial result of the reduction of MULTICUT to bounded treewidth, and was obtained in collaboration with Christophe Paul and Anthony Perez [6]. The fact that a vertex cutset $Y$ could be obtained by iterative compression, a tool of [18], was brought to our attention by Sylvain Guillemot. Finally, we would like to thank Valentin Garnero who worked on this project as a part of his graduate research experience in June 2010 .

\section{PRELIMINARIES}

A vertex which sends a request is called a terminal. The number of requests sent by a terminal is its request degree. We study MULTICUT variants with additional constraints on the deleted edges. In the original MULTICUT problem, we can delete any set of $k$ edges, but in some more constrained versions we must delete a prescribed number of edges on some particular paths. The total number of deleted edges is called deletion allowance of the multicut problem. We will make extensive use of the term bounded which always implicitly means bounded in terms of the deletion allowance. Also, when speaking of FPT time, we always mean $O\left(f(d) n^{c}\right)$ where $c$ is a fixed constant and $d$ is the deletion allowance. Let us discuss further some of the operations we will often perform.

Reductions. These are computations where the output is a new instance which is equivalent to the original instance with respect to the existence of a solution. One of the most natural reductions concerns irrelevant requests, i.e. a request $x y$ such that every multicut of $R \backslash x y$ actually cuts $x$ from $y$, where $R$ is the set of requests. If one can certify that a request $x y$ is irrelevant, the reduction just consists in replacing $R$ by $R \backslash x y$. The difficulty is obviously to certify that a request is irrelevant. Another easy reduction is obtained if we can certify that there exists a multicut which does not separate two given vertices $u$ and $v$. In this case we simply contract $u$ and $v$. Reductions are easy to control since we can perform them freely provided that some invariant polynomial in $n$ decreases. For instance, request deletions can be performed at most $n^{2}$ times, and vertex contractions at most $n$ times.

Branchings. In our algorithm, we often have to decide if the multicut we are looking for is of a particular type, where the number of types is bounded. We will then say that we branch over all the possible cases. This means that to compute the result of the current instance, we run our algorithm on each case, in which we force the solution to be of each given type. The output is TRUE if at least one of the outputs returns TRUE. To illustrate this, in the case of a graph $G$ with two connected components $G_{1}$ and $G_{2}$, both containing requests, we would branch over $k-1$ instances, 
depending of the number of edges (between 1 and $k-1$ ) that we delete from $G_{1}$. This simple branching explains why we can focus on connected graphs.

Invariants. To prove that the total number of branches is bounded, we show that some invariant is modified at each branching step, and that the number of times that this invariant can be modified is bounded. We usually have several invariants ordered lexicographically. In other words, we have different invariants which we want to increase or decrease and can take a bounded number of values. These invariants are ordered, there is a primary invariant, a secondary, etc. Each branching must improve our invariant, i.e. the first invariant (with respect to priority order) which is changed by the branching must be modified according to the preference, increase or decrease, that we specified for it. For instance the primary invariant could be the number of deleted edges, which we want to decrease and the secondary invariant could be the connectivity of $G$, which we want to increase. If we can decrease the number of deleted edges we do so even if the connectivity of the graph decreases. Also, if a branching increases connectivity and leaves the number of deleted edges unchanged, we improve our invariant.

\section{CONNECTIVITY IN FPT TIME}

Dealing with minimum cuts can be done in polynomial time with usual flow techniques. However, dealing with $k$ edge cuts when $k$ is some fixed value larger than the optimum is more difficult. We develop here some tools to deal in FPT time with bounded cuts.

\subsection{Enumerating cuts in FPT time}

The results in this subsection are of the same flavor as in [4], [6], [16] and [20]. Due to space restriction, we omit their proofs.

Let $G$ be a connected graph on $n$ vertices with a particular vertex $x$ called root. We deal in this part with cuts, i.e. bipartitions of the vertex set of $G$. To fix one side of our cuts, we define a cut as a subset of vertices $S$ containing $x$. The border of $S$ is the set of edges of $G$ with exactly one endpoint in $S$. We denote it by $\Delta(S)$. Its cardinality is denoted by $\delta(S)$. Recall that the function $\delta$ is submodular, i.e. $\delta(A)+\delta(B) \geq \delta(A \cap B)+\delta(A \cup B)$. Given a subset $Y$ of vertices of $V(G)$, we denote its complement $V(G) \backslash Y$ by $\bar{Y}$. A cut $S$ is a left cut if every cut $T \subsetneq S$ satisfies $\delta(T)>\delta(S)$. Note that every cut $S$ contains a left cut $S^{\prime}$ with $\delta\left(S^{\prime}\right) \leq \delta(S)$

LEMma 1. Left cuts are closed under union.

LEMMA 2. If $S_{1}, S_{2}$ are distinct left cuts, $\delta\left(S_{1} \cup S_{2}\right)<$ $\max \left(\delta\left(S_{1}\right), \delta\left(S_{2}\right)\right)$.

A cut $S$ is indivisible if $G \backslash S$ is connected, otherwise it is divisible.

LEMma 3. If $S$ is a divisible left cut and $Y$ is a connected component of $G \backslash S$, the cut $\bar{Y}$ is an indivisible left cut with $\delta(\bar{Y})<\delta(S)$.

COROLlary 4. Every indivisible cut $S$ contains an indivisible left cut $S^{\prime}$ with $\delta\left(S^{\prime}\right) \leq \delta(S)$.

Given a vertex $y$, an $x y$-cut is a cut $S$ such that $y \notin S$. We denote by $C_{k}^{y}$ the set of indivisible left $x y$-cuts with border $k$. We also denote by $C_{<k}^{y}\left(\right.$ resp. $\left.C_{<k}^{y}\right)$ the union of $C_{i}^{y}$ for $i<k$ (resp. for $i \leq k$ ).
THEOREM 5. The set $C_{\leq k}^{y}$ has size at most $k$ ! and can be computed in FPT time.

The value $k$ ! in Theorem 5 can actually be improved to $4^{k}[3,19]$.

\subsection{Irrelevant Requests}

We denote by $C_{k}$ the union of $C_{k}^{y}$ for all vertices $y$ of $G$, by $C_{<k}$ the union of $C_{<k}^{y}$ for all vertices $y$ of $G$, and by $C_{\leq k}$ the union of $C_{\leq k}^{y}$ for all vertices $y$ of $G$.

THEOREM 6. Every set $K$ with at least $\alpha\left(k, k^{\prime}\right):=k^{\left(\begin{array}{c}k+2 \\ 2\end{array}\right)-1}$ vertices of $G$ contains a subset $K^{\prime}$ of size $k^{\prime}$ such that every left cut $S$ with $\delta(S) \leq k$ satisfies either $S \cap K^{\prime}=\emptyset$ or $\left|K^{\prime} \backslash S\right| \leq k$. The set $K^{\prime}$ can be computed in FPT single exponential time.

Proof. Observe that the result trivially holds when $k^{\prime} \leq$ $k$. So we can assume that $k^{\prime}>k$. Consider a graph $G$ rooted at $x$. We prove the result by induction on $k$.

For $k=1, \alpha\left(1, k^{\prime}\right)=k^{\prime 2}$. The complements of a left cut with a border of size 1 form a collection of disjoint sets of vertices, which induces a partition of $K$. There is either a class $K^{\prime}$ of this partition containing at least $\sqrt{|K|} \geq k^{\prime}$ elements, or a set $K^{\prime}$ of size at least $\sqrt{|K|} \geq k^{\prime}$ which elements are chosen in different classes. In both cases $K^{\prime}$ satisfies the induction hypothesis.

Assume now that $k>1$. We distinguish two cases.

Assume that there exists an indivisible left cut $S$ with $\delta(S) \leq k$ and $|K \backslash S| \geq k^{\left(\begin{array}{c}k+1 \\ 2\end{array}\right)-1}$. By induction, we extract from $K \backslash S$ a subset $K^{\prime}$ with size $k^{\prime}$ such that every left cut $T$ with $\delta(T) \leq k-1$ satisfies either $T \cap K^{\prime}=\emptyset$ or $\left|K^{\prime} \backslash T\right| \leq k-1$. To conclude, let us consider a left cut $S^{\prime}$ with $\delta\left(S^{\prime}\right)=k$. If $S^{\prime}=S$, then $S \cap K^{\prime}=\emptyset$ by definition of $K^{\prime}$, hence we assume that $S^{\prime}$ is distinct from $S$. Observe that $K^{\prime} \backslash S^{\prime}$ is equal to $K^{\prime} \backslash\left(S \cup S^{\prime}\right)$. By Lemma $2, S \cup S^{\prime}$ is a left cut with border at most $k-1$, so $K^{\prime} \backslash S^{\prime}$ is either $K^{\prime}$ or has size at most $k-1$. Hence the conclusion of Theorem 6 holds.

Conversely, assume that all indivisible left cuts $S$ with $\delta(S) \leq k$ satisfy $|K \backslash S|<k^{\prime\left(\begin{array}{c}k+1 \\ 2\end{array}\right)-1}$. Let us form a graph $H$ with vertex set $K$ and where $v v^{\prime}$ is an edge when there exists an indivisible left cut $S$ with $\delta(S) \leq k$ such that $\left\{v, v^{\prime}\right\} \cap$ $S=\emptyset$. The degree of a vertex $v$ of $H$ is less than $d:=$ $k^{\prime\left(\begin{array}{c}k+1 \\ 2\end{array}\right)-1} \cdot k$ !. Indeed, the number of indivisible left $x v$-cuts with border at most $k$ is at most $k$ !, and we have assumed that all indivisible left cuts $S$ with $\delta(S) \leq k$ satisfy $|K \backslash S|<$ $k^{\prime\left(\begin{array}{c}k+1 \\ 2\end{array}\right)-1}$. Note that $d$ is less than $k^{\left(\begin{array}{c}k+1 \\ 2\end{array}\right)-1} \cdot k^{\prime k}$ since $k^{\prime} \geq k$. There is a stable set $K^{\prime}$ in $H$ of size at least $\frac{|K|}{d} \geq k^{\prime}$ since $\left(\begin{array}{c}k+2 \\ 2\end{array}\right)-\left(\begin{array}{c}k+1 \\ 2\end{array}\right)-k=1$. Every indivisible left cut $S$ with $\delta(S) \leq k$ isolates at most one vertex of $K^{\prime}$ as $K^{\prime}$ is stable in $H$. Hence every left cut $S$ with $\delta(S) \leq k$ isolates at most $k$ vertices of $K^{\prime}$.

The induction hypothesis holds in both cases, which concludes the proof of Theorem 6 .

The next result is our key tool in reducing MULTICUT to graphs of bounded treewidth in [6], but the following easy argument is more straightforward. The proof of Theorem 4 of [6] implies that the following result holds with $h(\ell)=$ $\ell^{O(\ell)}$, and can be computed in time $\ell^{O(\ell)} n^{c}$. 
THEOREM 7. Every set $K$ with at least $h(\ell):=\ell .2^{\ell !}+1$ vertices of $G$ contains a vertex $y$ such that every cut $S$ with $\delta(S)+|S \cap K| \leq \ell$ is such that $y \notin S$. Moreover, $y$ is computed in FPT time.

In other words, vertex $y$ verifies the following: whenever the deletion of a set of $a$ edges isolates $x$ from all but $b$ elements of $K$, with $a+b \leq \ell$, then the vertex $y$ is also isolated from $x$.

Proof. We create a new vertex $z$ joined to all the vertices of $K$ to form a new graph $G^{\prime}$. In this proof we change our point of view and consider that $z$ is our root vertex for $G^{\prime}$, i.e. $z$ plays for $G^{\prime}$ the same role as $x$ plays for $G$. In $G^{\prime}$, the set $C$ of indivisible left $z x$-cuts with border at most $\ell$ has size at most $\ell$ ! by Theorem 5 . Hence, since the size of $K$ is at least $\ell .2^{\ell !}+1$, there exists a subset $T$ of $K$ of size at least $\ell+1$ such that for every cut $S$ in $C$, we have either $T \subseteq S$ or $T \cap S=\emptyset$. We compute such a set $T$ in FPT time. We then pick $y$ in $T$. Let us prove that $y$ satisfies the conclusion of Theorem 7 .

In the graph $G$, consider a set $A$ of $a$ edges which isolates $x$ from all the elements of $K$ save a subset $B$ of size $b$ with $a+b \leq \ell$. Let $F$ be the set of edges $A \cup\{z b: b \in B\}$ of $G^{\prime}$. Note that $F$ is a $z x$-edge cut. We denote by $X$ the component of $x$ in $G^{\prime} \backslash F$. Since $V\left(G^{\prime}\right) \backslash X$ is an indivisible $z x$-cut with border at most $\ell$, it contains by Corollary 4 a indivisible left $z x$-cut $S$ with border at most $\ell$, in other words $S$ belongs to $C$.

Let us first observe that the set $T$ cannot be disjoint from $S$. Indeed $T$ has size $\ell+1$ and each vertex of $T$ is adjacent to $z$, thus the border of $S$ would exceed $\ell$. Hence $T$ is included in $S$, thus the set of edges $A$ isolates $T$ from $x$, and in particular $y$ from $x$. This concludes our proof.

COROLlARY 8. In a multicut problem instance with deletion allowance $k$, the maximum request degree can be reduced to at most $h(k+1)$ in FPT time.

Proof. Let $x$ be a vertex which sends requests to all vertices of $K$, where $|K| \geq h(k+1)$. By Theorem 7 , there is a vertex $y$ of $K$ such that every subset $S$ containing $x$ such that $\delta(S)+|S \cap K| \leq k+1$ is such that $y \notin S$. We simply delete the request $x y$ from the set of requests. Indeed, let $F$ be a multicut of size at most $k$ of this reduced instance. Let $S$ be the component of $x$ in $G \backslash F$. Since $F$ is a multicut, no element of $K \backslash y$ belongs to $S$. Moreover $\delta(S) \leq k$ since at most $k$ edges are deleted. Thus $\delta(S)+|S \cap K|$ is at most $k+1$, which implies that $y \notin S$. In other words, even if we do not require to cut $x$ from $y$, any multicut of the reduced instance will cut the request $x y$. Therefore the request $x y$ can be deleted from $R$.

\subsection{Cherry Reduction}

An $x$-cherry, or simply cherry is a connected induced subgraph $C$ of $G$ with a particular vertex $x$ called attachment vertex of $C$ such that there is no edge from $C \backslash x$ to $G \backslash C$ and no request has its two terminals in $C \backslash x$. In other words the only requests inside an $x$-cherry have origin $x$. Note that we can always assume that the restriction of a multicut to an $x$-cherry $C$ is the border of a left cut of $C$. If $u \in C \backslash x$, a request $u v \in R$ is irrelevant if every multicut $F$ with at most $k$ edges of the reduced instance on $R \backslash u v$ and such that $F \cap C$ is the border of a left cut in $C$ actually separates $u$ from $v$.
THEOREM 9. Let $C$ be an $x$-cherry of a instance with deletion allowance $k$. We can find in FPT time a set $K(C)$ of at most $b(k):=h(k+1) \cdot \alpha(k, h(2 k+1))=k^{O\left(k^{3}\right)}$ terminals in $C \backslash x$, such that if $F$ is a set of at most $k$ edges which cuts all requests with one endpoint in $K(C)$ and such that $F \cap C$ is the border of a left cut, then $F$ actually cuts all requests with an endpoint in $C \backslash x$.

The proof's ideas are similar to the three previous proofs, and we omit it due to space restrictions.

Let $C$ be a cherry of a graph $G$ with deletion allowance $k$. A subset $\mathcal{L}$ of the edges of $C$ is active when, if a multicut $F$ of size at most $k$ exists, then there exists a multicut $F^{\prime}$ of size at most $|F|$ such that $F^{\prime} \backslash C=F \backslash C$ and $F^{\prime} \cap C \subseteq \mathcal{L}$. When the set $\mathcal{L}$ is clear from the context, we say by extension that edges of $\mathcal{L}$ are active.

Lemma 10. Let $C$ be an $x$-cherry of a graph $G$ with deletion allowance $k$, and let $K$ be the set of all terminals of $C \backslash x$. Let $\mathcal{L}(C)$ be the union of all borders of cuts of $C_{\leq k}^{y}$, where $y \in K$. Then $\mathcal{L}(C)$ is active, and has size at most $k|K| k !$.

Proof. Assume that $F$ is a multicut with size at most $k$. Let $S$ be the component of $x$ in $C \backslash F$. Let $T$ be a left cut with $T \subseteq S$ and $\delta(T) \leq \delta(S)$. If a component $U$ of $\bar{T}$ does not intersect $K$, then $\bar{F} \backslash \Delta(U)$ is still a multicut. Hence we can assume that all components $U$ of $\bar{T}$ intersect $K$, in which case $\Delta(U) \in C_{\leq k}^{y}$ for some $y$ in $K$, hence $\Delta(T)$ is included in $\mathcal{L}(C)$. The set $F^{\prime}=(F \backslash C) \cup \Delta(T)$ is a multicut, and the size bound for $\mathcal{L}(C)$ follows from Theorem 5 .

THEOREM 11. Let $H_{1}, H_{2}, \ldots, H_{p}$ be $x$-cherries of a graph $G$ with deletion allowance $k$ such that $H_{1} \backslash x, H_{2} \backslash x, \ldots, H_{p} \backslash x$ are pairwise disjoint. Assume that for every $i, U_{i}:=H_{1} \cup$ $\cdots \cup H_{i}$ is a cherry. Then every $U_{i}$ has a bounded active set $\mathcal{L}_{i}$ such that $\mathcal{L}_{j} \cap U_{i} \subseteq \mathcal{L}_{i}$ whenever $i \leq j$.

Proof. By Theorem 9, we can reduce the set of terminals in $U_{1}$ to a bounded set $K_{1}$. The set $\mathcal{L}_{1}=\mathcal{L}\left(U_{1}\right)$ is bounded and active by Lemma 10 . The requests of $C_{1} \backslash K_{1}$ are irrelevant in $U_{2}$ since they are irrelevant in $U_{1}$, hence we can assume that Theorem 9 applied to $U_{2}$ yields a set of terminals $K_{2} \subseteq K_{1} \cup C_{2}$. Let $\mathcal{L}_{2}$ be the active edges associated to $K_{2}$. Note that if an edge $e \in \mathcal{L}_{2}$ is in $U_{1}$, it must belong to some $C_{\leq k}^{y}$ for some $y \in K_{2} \cap U_{1}$. Since $K_{2} \subseteq K_{1} \cup C_{2}$, we have $y \in K_{1}$, hence $e \in \mathcal{L}_{1}$, which is the property we are looking for. We extract $K_{3}$ from $K_{2} \cup C_{3}$, and iterate our process to form the sequence $\mathcal{L}_{i}$.

\section{REDUCING TO COMPONENT MULTI- CUT}

Let $G=(V, E)$ be a connected graph, and $R$ be a set of requests. A vertex-multicut $Y$ is a subset of $V$ such that every $x y$-path of $G$ where $x y \in R$ contains an element of $Y$. Let $A$ be a connected component of $G \backslash Y$. We call $Y$-component, or component, the union of $A$ and its set of neighbors in $Y$. Let $C$ be a component, the vertices of $C \cap Y$ are the attachment vertices of $C$.

\subsection{Component Multicut}

Here is our first intermediate problem. 
COMPONENT MULTICUT:

Input: A connected graph $G=(V, E)$, a vertex-multicut $Y$, a set of requests $R$, a set $I$ of $q$ integers such that:

(i) There are $q Y$-components $G_{1}, \ldots, G_{q}$ with two attachment vertices $x_{i}, y_{i}$. The other components have only one attachment vertex.

(ii) Every $G_{i}$ has a $x_{i} y_{i}$-path $P_{i}$ called backbone of $G_{i}$. Moreover, the deletion of every edge of $P_{i}$ decreases the edge connectivity in $G_{i}$ between $x_{i}$ and $y_{i}$.

(iii) The set $I$ consists of $q$ non-negative integers $f_{1}, \ldots, f_{q}$ such that $f_{1}+\cdots+f_{q} \leq k-q$.

Parameter: $k$.

Output: TRUE if there exists a multicut $F$ such that:

(i) every path $P_{i}$ contains exactly one edge of $F$,

(ii) every $G_{i}$ contains exactly $1+f_{i}$ edges of $F$,

(iii) the solution $F$ splits $Y$, i.e. the connected components of $G \backslash F$ contain at most one vertex of $Y$.

Otherwise, the output is FALSE.

We call $Y$ a cutset, i.e. a vertex-multicut which must be split by the solution $F$. The edges of $G$ which do not belong to the backbones are called free edges. The backbone $P_{i}$, in which only one edge is deleted, is the crucial structure of $G_{i}$. Indeed, the whole proof consists of modifying $G_{i}$ step by step to finally completely reduce it to the backbone $P_{i}$. Here $f_{i}$ is the number of free edges one can delete in $G_{i}$. Observe that $k-q-f_{1}-\cdots-f_{q}$ free edges can be deleted in components with one attachment vertex. Our first reduction is the following:

THEOREM 12. MULTICUT can be reduced to COMPONENT MULTICUT in FPT time.

The remaining of Section 4 is devoted to the proof of Theorem 12. We first construct a cutset $Y$. Then we prove that we can reduce to $Y$-components with one or two attachment vertices. Finally, we show that we can assume that every component with two attachment vertices has a path in which exactly one edge is chosen in the solution. This is our backbone.

\subsection{The cutset $Y$.}

We use iterative compression to prove the existence of a vertex-multicut of size $k+1$, as was done in [18].

LEMMA 13. MULTICUT can be solved in time $O\left(f(k) n^{c}\right)$ if MULTICUT given a vertex multicut of size at most $k+1$ can be solved in time $O\left(f(k) n^{c-1}\right)$.

Proof. By induction on $n$, we solve MULTICUT in time $f(k)(n-1)^{c}$ on $G-v$, where $v \in V(G)$. If the output is FALSE, we return FALSE, otherwise the output is a multicut $F$ of size at most $k$. Let $X$ be a vertex cover of $F$ of size at most $k$. Thus $X \cup\{v\}$ is a vertex-multicut of our instance, so we solve MULTICUT in time $f(k) n^{c-1}+f(k)(n-1)^{c}$ which is at most $f(k) n^{c}$.

So we can assume that we have a vertex-multicut $Y$ of size at most $k+1$.
Lemma 14. We can assume that the solution $F$ splits $Y$.

Proof. To any solution $F$ is associated the partition of $G \backslash F$ into connected components. In particular this induces a partition of $Y$. We branch over all possible partitions of $Y$. In a given branch, we simply contract the elements of $Y$ belonging to the same part of the partition corresponding to the branch.

During the following reduction proof, the size of the set $Y$ will never decrease. Since one needs $k+1$ edges to separate $k+2$ vertices, the size of $Y$ cannot exceed $k+1$, otherwise we return FALSE. Hence our primary invariant is the size of $Y$, and we immediately conclude if we can make it increase.

\subsection{Reducing Attachment Vertices}

Our second invariant, which we intend to maximize, is the number of $Y$-components with at least two attachment vertices. This number cannot exceed $k$, since any solution splits $Y$. Our third invariant is the sum of the edge connectivity between all pairs of vertices of $Y$, which we want to increase. This invariant is bounded by $k\left(\begin{array}{c}|Y| \\ 2\end{array}\right)$ since the connectivity between two elements of $Y$ is at most $k$. Note that this invariant never decreases when we contract vertices.

Lemma 15. If $C$ is a $Y$-component with at least three attachment vertices, we improve our invariant.

Proof. Let $x, y, z$ be attachment vertices of $C$. Let $\lambda$ be the edge-connectivity between $x$ and $y$ in $C$. Let $P_{1}, \ldots, P_{\lambda}$ be a set of edge-disjoint $x y$-paths. A critical edge is an edge which belongs to some $x y$-edge cut of size $\lambda$. Note that every critical edge belongs to some path $P_{i}$. A slice of $C$ is a connected component of $C$ minus the critical edges. Given a vertex $v$ of $C$, the slice of $v$, denoted by $S L(v)$, is the slice of $C$ containing $v$. Let $B(z)$ be the border of $S L(z)$, i.e. the set of vertices of $S L(z)$ which are incident to a critical edge. Note that $B(z)$ intersects every path $P_{i}$ on at most two vertices, namely the leftmost vertex of $P_{i}$ belonging to $S L(z)$ and the rightmost vertex of $P_{i}$ belonging to $S L(z)$. In particular, $B(z)$ has $b$ vertices, where $b \leq 2 \lambda$.

We branch over $b+1$ choices to decide whether one of the $b$ vertices of $B(z)$ belongs to a component of $G \backslash F$ (where $F$ is our solution) which does not contain a vertex of $Y$. When this is the case, the vertex is added to $Y$, hence we increase our primary invariant. In the last branch, all the vertices of $B(z)$ are connected to a vertex of $Y$ in $G \backslash F$. We branch again over all mappings $f$ from $B(z)$ into $Y$. In each branch, the vertex $v \in B(z)$ is connected to $f(v) \in Y$ in $G \backslash F$. Hence we can contract every vertex $v \in B(z)$ to the vertex $f(v)$ of $Y$. This gives a new graph $G^{\prime}$. We denote by $S^{\prime}$ the subgraph $S L(z)$ after contraction of the vertices of $B(z)$. Observe that $S^{\prime}$ is a $Y$-component of $G^{\prime}$.

If $x$ and $y$ belong to $S^{\prime}$, the edge connectivity between $x$ and $y$ has increased. Indeed, there is now a path $P$ joining $x$ to $y$ inside $S^{\prime}$, in particular $P$ has no critical edge. Thus the connectivity between $x$ and $y$ has increased, so our invariant has improved. We assume without loss of generality that $x$ does not belong to $S^{\prime}$.

If $S^{\prime}$ contains an element of $Y$ distinct from $z$, then $S^{\prime}$ is a $Y$-component with at least two attachment vertices. Moreover, there exists a path $P$ in $C \backslash S^{\prime}$ from $x$ to $B(z)$. Hence we have created an extra $Y$-component with at least two attachment vertices in $G^{\prime}$, which improves our second invariant. 
In our last case, $S^{\prime}$ only intersects $Y$ on $z$. Therefore $B(z)$ is entirely contracted to $z$. In particular $z$ is now incident to a critical edge $e$. So there exists an $x y$-cut $A$ with $\delta(A)=\lambda$ and $e \in \Delta(A)$. Without loss of generality, we assume that $z \notin A$ (otherwise we consider the $y x$-cut $\bar{A}$ ). We denote by $B$ the vertices of $\bar{A}$ with a neighbor in $A$. In particular, $B$ contains $z$, has size at most $\lambda$, and every $x y$-path in $C$ contains a vertex of $B$. Let us denote by $L$ the set $A \cup B$ and by $R$ the set $\bar{A}$. Note that $L \cap R=B$. We now branch to decide in which components of $G \backslash F$ the elements of $B$ are partitioned. If an element of $B$ is not connected to $Y$ in $G \backslash F$, we improve our invariant. If each element of $B$ is contracted to a vertex of $Y$, both $L$ and $R$ in the contracted graph are $Y$-components with at least two attachment vertices (respectively $\{x, z\}$ and $\{y, z\}$ ). We again improve our invariant.

\subsection{Backbones}

We now assume that every component has at most two attachment vertices. Let $G_{1}, \ldots, G_{q}$ be the components of $G$ with two attachment vertices. We denote by $\lambda_{i}$ the edge connectivity of $G_{i}$ between its two attachment vertices $x_{i}, y_{i}$. Recall that our third invariant is just the sum of the $\lambda_{i}$.

LEMma 16. We can assume that $x_{i}$ and $y_{i}$ have degree $\lambda_{i}$ in $G_{i}$.

Proof. Let $A$ be the unique left $x_{i} y_{i}$-cut with $\delta(A)=\lambda_{i}$ in the graph $G_{i}$ rooted in $x_{i}$. Let $B$ be the set of vertices of $A$ with a neighbor in $\bar{A}$. We now branch to decide how the components of $G \backslash F$ partition $B$. If an element of $B$ is not connected to $Y$ in $G \backslash F$, we improve our invariant. If an element of $B$ is contracted to $y_{i}$, we increase $\lambda_{i}$. Hence all elements of $B$ are contracted to $x_{i}$. Therefore $A$ becomes an $x_{i}$-cherry, hence $A \backslash x_{i}$ is removed from $G_{i}$. The degree of $x_{i}$ inside $G_{i}$ is now exactly $\lambda_{i}$. We apply the same argument to reduce the degree of $y_{i}$ to $\lambda_{i}$.

We now branch over all partitions of $k$ into $k_{0}+k_{1}+\cdots+$ $k_{q}=k$, where $k_{i}$ is the number of edges of our solution chosen in $G_{i}$ when $i>0$, and $k_{0}$ is the number of edges chosen in the $y$-components for $y \in Y$.

LEMMA 17. Every component $G_{i}$ can be deleted or has a backbone.

Proof. If $k_{i} \geq 2 \lambda_{i}$, we simply delete in $G_{i}$ the edges incident to $x_{i}$ and $y_{i}$, reduce our parameter, and improve our invariant. So we can assume that $k_{i} \leq 2 \lambda_{i}-1$. Let $P_{1}, P_{2}, \ldots, P_{\lambda_{i}}$ be some edge-disjoint $x_{i} y_{i}$-paths. Our algorithm now branches $2 \lambda_{i}$ times, where the branches are called $B_{j}$ and $B_{j}^{\prime}$ for $j=1, \ldots, \lambda_{i}$. In the branch $B_{j}$, we assume that there is only one edge of our solution selected in $P_{j}$, and this edge belongs to an $x_{i} y_{i}$-cut of size $\lambda_{i}$. In the branch $B_{j}^{\prime}$, we assume that all the edges of our solution selected in $P_{j}$ are not critical. Let us show that any solution $F$ belongs to one of these branches. If $F$ does not belong to any branch $B_{j}^{\prime}$, this means that $F$ uses at least one critical edge in each $P_{j}$. But since $k_{i} \leq 2 \lambda_{i}-1$ some $P_{j}$ only intersects $F$ on one edge, which is therefore critical. Hence $F$ is a solution in the branch $B_{j}$. Thus this branching process is valid. In the branch $B_{j}$, we contract all non critical edges of $P_{j}$, therefore $P_{j}$ is the backbone we are looking for. In the branch $B_{j}^{\prime}$, we contract all critical edges of $P_{j}$, hence the connectivity $\lambda_{i}$ increases. We thus improve our invariant.

This concludes the proof of Theorem 12 .

\section{BACKBONE MULTICUT IS FPT}

\subsection{Backbone Multicut}

We introduce here the problem BACKBONE MULTICUT, which is a generalization of COMPONENT MULTICUT. Our goal is to show that BACKBONE MULTICUT is solvable in FPT time, which implies that COMPONENT MULTICUT is FPT, which in turns implies that MULTICUT is FPT thanks to Theorem 12.

BACKBONE MULTICUT:

Input: A connected graph $G=(V, E)$, a set of half-requests $R$, a set $Y$ of vertices, a set $B$ of $q$ variables, a set $\mathcal{C}$ of clauses, a set $I$ of $q$ integers such that:

(i) $G$ has $q Y$-components called $G_{i}$ with two attachment vertices $x_{i}, y_{i} \in Y$, with $i=1, \ldots, q$. Moreover, $G_{i}$ has a backbone $P_{i}$ and the $x_{i}, y_{i}$-connectivity in $G_{i}$ is $\lambda_{i}$. Recall that the edges of $G$ which are not in backbones are called free edges.

(ii) The set $R$ contains half-requests, i.e. sets of triples $(u, y, v)$, informally meaning that vertex $u$ sends a request to vertex $v$ via $y$, where $y \in Y$. Also, $Y$ is a $u, v$-cut for every half-request $(u, y, v) \in R$.

(iii) The set $B$ contains $q$ integer-valued variables $c_{1}, \ldots, c_{q}$. Each variable $c_{i}$ corresponds to the deletion of one edge in the backbone $P_{i}$. Formally, if the edges of $P_{i}$ are $e_{1}, \ldots, e_{\ell_{i}}$, ordered from $x_{i}$ to $y_{i}$, the variable $c_{i}$ can take all possible values from 1 to $\ell_{i}$, and $c_{i}=r$ means that we delete the edge $e_{r}$ in $P_{i}$.

(iv) The clauses in $\mathcal{C}$ have four possible types: $\left(c_{i} \leq a \Rightarrow\right.$ $\left.c_{j} \leq b\right)$, or $\left(c_{i} \leq a \Rightarrow c_{j} \geq b\right)$, or $\left(c_{i} \geq a \Rightarrow c_{j} \geq b\right)$, or $\left(c_{i} \geq a \Rightarrow c_{j} \leq b\right)$.

(v) The set $I$ consists of $q$ non-negative integers $f_{1}, \ldots, f_{q}$ summing to a value at most $k$. Each integer $f_{i}$ corresponds to the number of free edges of the solution which are chosen in $G_{i}$.

Parameter: $k$.

Output: TRUE if:

(i) There exists an assignment of the variables of $B$ which satisfies $\mathcal{C}$

(ii) There exists a subset $F$ of at most $k$ free edges of $G$, which contains $f_{i}$ free edges in $G_{i}$ for $i=1, \ldots, q$.

(iii) The union $F^{\prime}$ of $F$ and the backbone edges corresponding to the variables of $B$ splits $Y$ and intersects every half-request of $R$, i.e. for every half-request $(u, y, v) \in R$ every path between $u$ and $v$ containing $y$ intersects $F^{\prime}$.

Otherwise, the output is FALSE.

Note that the deletion allowance of BACKBONE MULTICUT is $k+q$. COMPONENT MULTICUT directly translates into BACKBONE MULTICUT with an empty set of clauses, and where each request is simulated by one or two half-requests. This section is devoted to the proof of the following result.

THEOREM 18. BACKBONE MULTICUT can be solved in FPT time. 


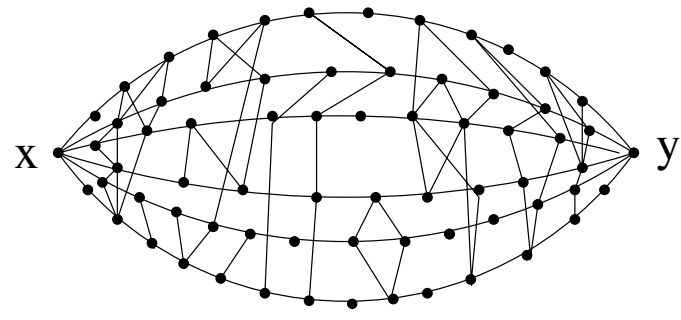

Figure 1: A component in a BACKBONE MULTICUT instance. The path at the bottom is the backbone. Note that each edge of the backbone does indeed belong to a $\lambda$-cut.

\subsection{Invariants}

Our primary invariant is the sum of the $f_{i}$, which starts with value at most $k$ and is non-negative. Any branch in which we can decrease it will be considered solved. Our secondary invariant is the sum of the $\lambda_{i}-1$, called the free connectivity, which we try to increase. Observe that this invariant is bounded above by $k$. For our last invariant, recall that the slice $S L(v)$ of some vertex $v$ in a component $G_{i}$ is the connected component containing $v$ of $G_{i}$ minus its critical edges, i.e. edges of $\lambda_{i}$-cuts. Observe that since the edges of $P_{i}$ are critical, the slices of distinct vertices in $P_{i}$ do not intersect. The slice connectivity of a vertex $v$ in $P_{i}$ is the $x_{i} y_{i}$-edge-connectivity of $G_{i} \backslash S L(v)$. We denote it by $s c(v)$. For instance, if the set of neighbors of $v$ intersect every $x_{i} y_{i}$ path in $G_{i} \backslash P_{i}$ then we have $s c(v)=0$. Conversely, if $v \in P_{i}$ has only neighbors in $P_{i}$, we have $s c(v)=\lambda_{i}-1$. The slice connectivity $s c_{i}$ of $G_{i}$ is the maximum of $s c(v)$, where $v \in P_{i}$. Our third invariant is the sum $s c$ of the $s c_{i}$, for $i=1, \ldots, q$, and we try to minimize this invariant. Observe that at any step, $s c$ is at most $k$.

Our goal is to show that we can always improve our invariant, or conclude that $\lambda_{i}=1$ for all $i$. We consider a component $G_{i}$ with $\lambda_{i}>1$, say $G_{1}$. To avoid cumbersome indices, we assume that the attachment vertices of $G_{1}$ are $x$ and $y$, and that their edge-connectivity is denoted by $\lambda$ instead of $\lambda_{1}$. Moreover, we still denote by $P_{1}$ the backbone of $G_{1}$, but we assume (against our previous notations) that $P_{1}, P_{2}, \ldots, P_{\lambda}$ is a set of edge-disjoint $x y$-paths in $G_{1}$. We visualize $x$ to the left and $y$ to the right (see Figure 1). Hence when we say that a vertex $u \in P_{i}$ is to the left of some $v \in P_{i}$, we mean that $u$ is between $x$ and $v$ on $P_{i}$.

\subsection{Contracting Edges}

In our proof, we contract edges of the backbone and also free edges which are not critical. At each step, we always preserve the fact that the edges of the backbone are critical.

When contracting an edge of the backbone $P_{1}$, we need to modify several parameters. Assume that the edges of $P_{1}$ are $e_{1}, \ldots, e_{\ell}$. The variable $c_{1}$ represents the edge of $P_{1}$ which is deleted in our multicut. Now assume that the edge $e_{i}=v_{i} v_{i+1}$ is contracted. All the indices of the edges which are at least $i+1$ are decreased by one. All the constraints associated to the other backbones are not affected by the transformation. However, each time a clause contains a literal $c_{1} \geq j$, where $j>i$, this literal must be replaced by $c_{1} \geq j-1$. Similarly each occurrence of $c_{1} \leq j^{\prime}$ for $j^{\prime} \geq i$ must be replaced by $c_{1} \leq j^{\prime}-1$. If a set of edges is contracted, we perform the contractions one by one.
The collection of paths $P_{2}, \ldots, P_{\lambda}$ can be affected during our contractions since it can happen that a path $P_{i}$ with $i \geq 2$ contains both endpoints of a contracted edge $u v$. In such a case, we remove from $P_{i}$ the loop formed by the contraction, i.e. the subpath of $P_{i}$ between $u$ and $v$. We thus preserve our path collection.

\subsection{Choosing a Stable Edge}

Let $v$ be a vertex of $P_{1}$. The $\operatorname{tag}$ of $v$ is the subset $t(v):=$ $\left\{i: P_{i} \cap S L(v) \neq \emptyset\right\}$, i.e. the set of indices of the paths intersecting the slice of $v$. Note that $t(v)$ contains 1 . Observe also that the slice connectivity of $G_{1}$ is the maximum of $\lambda-|t(v)|$, where $v$ belongs to $P_{1}$. By extension, the tag of an edge $v_{i} v_{i+1}$ of the backbone $P_{1}$ is the ordered pair $\left(t\left(v_{i}\right), t\left(v_{i+1}\right)\right)$. When speaking of an $X Y$-edge, we implicitly mean that its tag is $(X, Y)$. In particular, the edge of $P_{1}$ which is selected in our solution has a given tag. We branch over the possible choices for the tag $X Y$ of the deleted edge of $P_{1}$. Let us assume that the chosen edge has tag $X Y$.

Lemma 19. If $X \neq Y$, we improve our invariant.

Proof. Since only one edge is cut in the backbone, we can contract all the edges of $P_{1}$ with tags different from $X Y$. Observe that when contracting some $U V$-edge of $P_{1}$, the tag of the resulting vertex contains $U \cup V$ since the slice of the resulting vertex contains the union of both slices (it can actually be larger). After our contraction, all the edges of $P_{1}$ between two consecutive occurrences of $X Y$-edges are contracted, hence the tag of every vertex of $P_{1}$ now contains $X \cup Y$. In particular, the slice connectivity of $G_{1}$ decreases while the free connectivity is unchanged. Thus our invariant improved.

Therefore we may assume that we choose an $X X$-edge in our solution. Let us contract all the edges of $P_{1}$ which are not $X X$-edges. By doing so, we now have that the tag of every vertex of $P_{1}$ contains $X$. After this contraction, our instance is modified, hence we have to branch again over the choice of the tag of the edge chosen in our solution. Any choice different from $X X$ increases the slice connectivity. Hence we can still assume that the tag of the chosen edge is $X X$.

The slice connectivity of $G_{1}$ is $\lambda-|X|$. An $X X$-edge $u v$ of the backbone is unstable if, when contracting $u v$, the tag of the vertex $u=v$ increases (i.e. strictly contains $X)$. Otherwise $u v$ is stable. We branch on the fact that the chosen $X X$-edge is stable or unstable.

LEMmA 20. If the chosen $X X$-edge is unstable, we improve the invariant.

The proof of Lemma 20 and of the following Lemma 21 are omitted due to space restrictions, but are easily obtained via simple branching.

\subsection{Contracting Slices}

In this part, we assume that the chosen edge of $P_{1}$ is a stable $X X$-edge. A vertex $v$ of $P_{1}$ is full if $v$ belongs to every $P_{i}$, where $i \in X$ (see Figure 2). Our goal in this subsection is to show that we can reduce to the case where $X=\{1, \ldots, \lambda\}$. By the previous section, any branching increasing the tag of the chosen edge would improve the invariant. So we assume that in all our branchings, the chosen edge is still a stable $X X$-edge. 


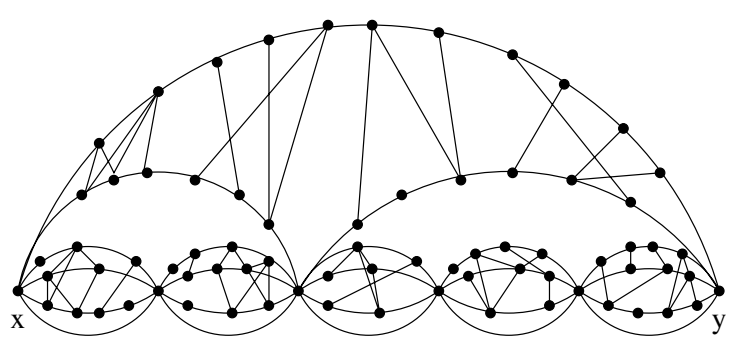

Figure 2: All backbone vertices of this component are full, where $X=\{1,2,3,4\}$, with the backbone $P_{1}$ at the bottom.

LEMMA 21. We can assume that all backbone vertices are full.

Note that after performing the reduction of Lemma 21, if $v_{i} v_{i+1}$ is a stable $X X$-edge, then for every vertex $w \in P_{j}$, with $j \in X$, which lies between $v_{i}$ and $v_{i+1}$ in $P_{j}$, every $w Y$ path contains $v_{i}$ or $v_{i+1}$. In particular, if $X=\{1, \ldots, \lambda\}$ then $v_{i}$ and $v_{i+1}$ are $x y$-cut vertices in $G_{1}$.

LEMma 22. We can assume that $X=\{1, \ldots, \lambda\}$.

In other words, we can reduce to the case where every vertex of $P_{1}$ is a cutvertex of $G_{1}$.

Proof. Assume that $X$ is not equal to $\{1, \ldots, \lambda\}$. We show that we can partition our component $G_{1}$ into two components $G_{1}^{1}$ and $G_{1}^{2}$. This partition leaves the freeconnectivity unchanged, but decreases the slice connectivity. A vertex $v_{i}$ of the backbone $P_{1}$ is left clean if the edge $v_{i-1} v_{i}$ of $P_{1}$ is a stable $X X$-edge, but the edge $v_{i} v_{i+1}$ of $P_{1}$ is not. It is right clean if the edge $v_{i} v_{i+1}$ is a stable $X X$ edge, but the edge $v_{i-1} v_{i}$ is not. Finally, $v_{i}$ is clean if both $v_{i-1} v_{i}$ and $v_{i} v_{i+1}$ are stable $X X$-edges. When enumerating all left clean and right clean vertices from left to right, we obtain the sequence of distinct vertices $r_{1}, l_{1}, r_{2}, l_{2}, \ldots, r_{p}, l_{p}$ where the $r_{i}$ are right clean and the $l_{i}$ are left clean vertices. Observe that $x$ and $y$ do not appear in the sequence since their tags are $\{1, \ldots, \lambda\}$. Let us consider a pair $r_{i}, l_{i}$. We say that a vertex $v$ of $G_{1}$ is between $r_{i}$ and $l_{i}$ if every path from $v$ to $x$ or $y$ intersects $\left\{r_{i}, l_{i}\right\}$. Let $B_{i}$ be the set of vertices which are between $r_{i}$ and $l_{i}$. Let $B$ be the union of $B_{i}$ for $i=1, \ldots, p$. Let $G_{1}^{1}$ be a copy of the subgraph induced by $B$ on $G_{1}$. Observe that $G_{1}^{1}$ has $p$ connected components, since $l_{i} \neq r_{i+1}$. We contract in $G_{1}^{1}$ the vertices $l_{i}$ and $r_{i+1}$, for all $i=1, \ldots, p-1$, hence making $G_{1}^{1}$ connected. We finally identify in $G_{1}^{1}$ the vertex $x$ with $r_{1}$ and $y$ with $l_{p}$. The backbone $P_{1}^{1}$ of $G_{1}^{1}$ simply consists of the edges of the original backbone. To construct $G_{1}^{2}$, we remove from $G_{1}$ all the vertices of $B$ which are not left clean or right clean vertices. Hence no stable $X X$-edge is left in $G_{1}^{2}$. We contract all the backbone edges of $G_{1}^{2}$. Formally, all the vertices between $x$ and $r_{1}$ are contracted to a vertex $w_{1}:=x$, more generally all the vertices between $l_{i}$ and $r_{i+1}$ are contracted to a new vertex called $w_{i+1}$, and finally all the vertices between $l_{p}$ and $y$ are contracted to $w_{p+1}:=y$. We now add the path $w_{1}, w_{2}, \ldots, w_{p+1}$ which is the backbone $P_{1}^{2}$ of $G_{1}^{2}$. We correlate the edges of the backbone of $G_{1}^{1}$ and $G_{1}^{2}$ by adding clauses implying that the chosen edge of $P_{1}^{2}$ is $w_{i} w_{i+1}$ if and only if the chosen edge of $P_{1}^{1}$ is between $r_{i}$ and $l_{i}$. We finally branch to split the number of free edges $f_{1}$ chosen

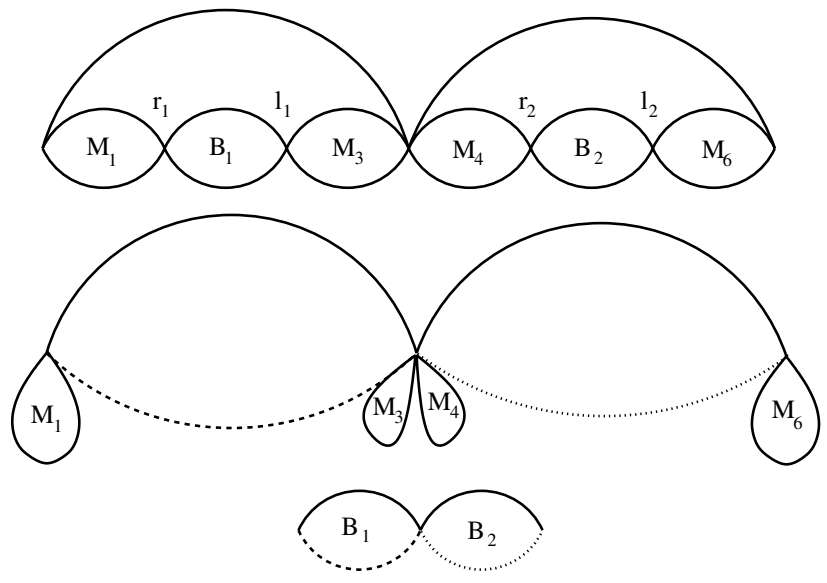

Figure 3: At the top, a component $G_{1}$ before transformation in the proof of Lemma 22. At the bottom, $G_{1}^{1}$ consists of $B_{1}$ and $B_{2}$, and $G_{1}^{2}$ is depicted in the center. The dashed edges in $G_{1}^{1}$ and $G_{1}^{2}$ are correlated, and similarly the dotted edges in $G_{1}^{1}$ and $G_{1}^{2}$ are correlated.

in $G_{1}$ into $f_{1}^{1}+f_{1}^{2}=f_{1}$, the respective free edges deleted in $G_{1}^{1}$ and $G_{1}^{2}$. Let us call $G^{\prime}$ the graph $G$ in which $G_{1}$ is replaced by $G_{1}^{1}$ and $G_{1}^{2}$. Note that the free edges of $G_{1}$ are partitioned into the free edges of $G_{1}^{1}$ and of $G_{1}^{2}$. Observe that the free-connectivity of $G$ and $G^{\prime}$ are equal. However, the slice connectivity has decreased in $G^{\prime}$, since its value is 0 in $G_{1}^{1}$ and strictly less than $\lambda-|X|$ in $G_{1}^{2}$. Indeed, for $i=1, \ldots, p-1$, the edge $l_{i} l_{i+1}$ is either unstable or the tag of one of its endpoints strictly contains $X$. Hence contracting all vertices between $l_{i}$ and $r_{i+1}$ strictly increases the tag of the resulting vertex in $G_{1}^{2}$. Hence we improve our invariant. Figure 3 gives an example of this transformation.

We now have to prove that there exists a multicut in $G^{\prime}$ if and only if there exists one in $G$ which uses a stable $X X$ edge. This comes from the following observation: Let $e=$ $v_{j} v_{j+1}$ be a stable $X X$-edge of $P_{1}$ between $r_{i}$ and $l_{i}$. Let $G_{e}$ be obtained from $G$ by deleting $e$, contracting $x$ with all the vertices of $P_{1}$ to the left of $v_{j}$, and contracting $y$ with all the vertices of $P_{1}$ to the right of $v_{j+1}$. Let $G_{e}^{\prime}$ be obtained from $G^{\prime}$ by deleting $e$ in $P_{1}^{1}$, deleting the edge $w_{i} w_{i+1}$ correlated to $e$ in $P_{1}^{2}$, contracting $x$ with all the vertices of $P_{1}^{1}$ to the left of $v_{j}$ and all the vertices of $P_{1}^{2}$ to the left of $w_{i}$, and contracting $y$ with all the vertices of $P_{1}^{1}$ to the right of $v_{j+1}$ and all the vertices of $P_{1}^{2}$ to the right of $w_{i+1}$. The key fact is that $G_{e}$ is equal to $G_{e}^{\prime}$. Hence the multicuts in $G$ and $G^{\prime}$ selecting the edge $e$ are in one to one correspondence.

The proof of Lemma 22 produces a new component, hence a new edge to be chosen in a backbone. This increases the deletion allowance by 1 , but the number of free edges has not increased.

\subsection{Reducing the Lemons}

We now assume that each vertex of the backbone $P_{1}$ of $G_{1}$ intersects all other paths $P_{i}$. Let $v_{i} v_{i+1}$ be an edge of the backbone $P_{1}$. The $v_{i}$-cherry $C_{i}$ is the set of all vertices $u$ of $G_{1}$ such that every $u Y$-path contains $v_{i}$.

The lemon $M_{i}$ of $v_{i} v_{i+1}$ is the set consisting of $v_{i}, v_{i+1}$ and of all vertices $u$ of $G_{1}$ which do not belong to a cherry and 


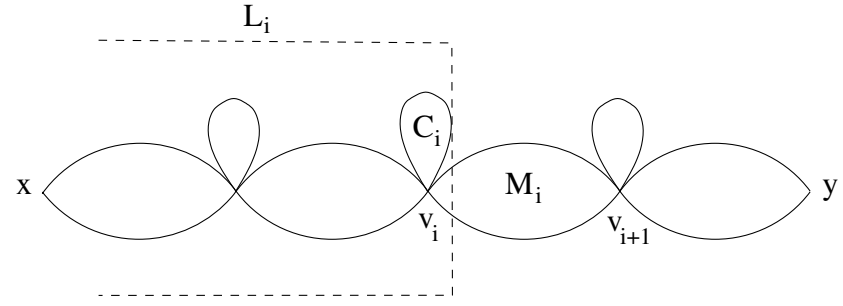

Figure 4: The left subgraph $L_{i}$ of $v_{i}$. The set $C_{i}$ is the $v_{i}$-cherry, and $M_{i}$ is the lemon of the backbone edge $v_{i} v_{i+1}$.

such that every $u x$-path in $G_{1}$ contains $v_{i}$ and every $u y$ path in $G_{1}$ contains $v_{i+1}$. Observe that when contracting $v_{i} v_{i+1}$, the lemon $M_{i}$ becomes part of the $v_{i}$-cherry, where $v_{i}$ denotes the resulting vertex. We denote by $L_{i}$ the union of all $C_{j}$ with $j \leq i$ and all $M_{j}$ with $j<i$. We call $L_{i}$ the left subgraph of $v_{i}$. Similarly, the right subgraph $R_{i}$ of $v_{i}$ is the union of all $C_{j}$ with $j \geq i$ and all $M_{j}$ with $j>i$. See Figure 4.

If a multicut $F$ selects the edge $v_{i} v_{i+1}$ in the backbone, then the vertices $x, v_{1}, \ldots, v_{i}$ all lie in the same connected component of $G \backslash F$. When these vertices $x, v_{1}, \ldots, v_{i}$ are contracted to $x$, the set $L_{i}$ becomes an $x$-cherry. Halfrequests through $y$ with an endpoint in $L_{i}$ are automatically cut since $F$ splits $Y$. Consider the terminals $T_{i}$ of halfrequests of $L_{i}$ which are routed via $x$. By Theorem 9 we can reduce $T_{i}$ to a bounded set of terminals $K_{i}$ (note that these half-requests become equivalent to usual requests, since $L_{i}$ is now an $x$-cherry). This motivates the following key definition.

By Lemma 10, we define $\mathcal{L}_{i}$ to be a bounded active set of edges in the $x$-cherry obtained from $L_{i}$ by contracting vertices $x, v_{1}, \ldots, v_{i}$. By Theorem 11 , we can compute such sets $\mathcal{L}_{i}$ so that $\mathcal{L}_{j} \cap L_{i} \subseteq \mathcal{L}_{i}$ when $i \leq j$.

Let us say that a multicut $F$ selecting $v_{i} v_{i+1}$ in $P_{1}$ is proper if $F \cap L_{i}$ is included in $\mathcal{L}_{i}$.

LEMMA 23. If there exists a multicut $F$ of size at most $k$ containing the backbone edge $v_{i} v_{i+1}$, then there is a proper multicut $F^{\prime}$ of size at most $k$ containing $v_{i} v_{i+1}$.

Proof. Consider a multicut $F$ containing $v_{i} v_{i+1}$. As the set $\mathcal{L}_{i}$ is active in the cherry obtained by contracting the path $x, v_{1}, \ldots, v_{i}$ in $L_{i}$, there exists a multicut $F^{\prime}$ of size $k$ such that $F^{\prime} \backslash L_{i}=F \backslash L_{i}$ and $F^{\prime} \cap L_{i} \subseteq \mathcal{L}_{i}$. Hence $F^{\prime}$ is proper and contains $v_{i} v_{i+1}$.

We denote by $\mathcal{L}$ the set of all subsets $F$ of size at most $k$ contained in some $\mathcal{L}_{i}$. We denote by $c$ the maximum size of a set $\mathcal{L}_{i}$. Note that $c$ is bounded in terms of $k$.

For two sets $F_{i} \subseteq \mathcal{L}_{i}$ and $F_{j} \subseteq \mathcal{L}_{j}$ with $j \geq i$, let us write $F_{i} \preceq F_{j}$ when $F_{j} \cap L_{i+1} \subseteq F_{i}$. Observe that $\preceq$ is a partial order. A subset $\mathcal{F}$ of $\mathcal{L}$ is correlated if:

- elements of $\mathcal{F}$ have the same size, and

- $\mathcal{F}$ is a chain for $\preceq$, i.e. if for every $F_{i}$ and $F_{j}$ in $\mathcal{F}$, with $F_{i} \subseteq \mathcal{L}_{i}, F_{j} \subseteq \mathcal{L}_{j}$ and $j \geq i$, we have $F_{j} \cap L_{i+1} \subseteq F_{i}$.

Lemma 24. There is a partition $\mathcal{F}_{1}, \mathcal{F}_{2}, \ldots, \mathcal{F}_{k(2 c)^{k}}$ of $\mathcal{L}$ into $k(2 c)^{k}$ correlated sets.
ProOF. Let us prove by induction on $\ell=0, \ldots, k$ that there exists no antichain for $\preceq$ in $\mathcal{L}$ consisting of $(2 c)^{\ell}+$ 1 sets of size at most $l$. This clearly holds for $\ell=0$. Assume that this holds for $\ell-1$. By contradiction, let $A=\left\{F_{1}, F_{2}, \ldots, F_{(2 c)^{\ell}+1}\right\}$ be an antichain of sets of size at most $\ell$. Let $t_{i}$ be an integer such that $F_{i} \subseteq \mathcal{L}_{t_{i}}$ for $i=1, \ldots,(2 c)^{\ell}+1$. We assume that the sets $F_{i}$ are enumerated in such a way that $t_{i} \leq t_{j}$ whenever $i \leq j$. The set $F_{1}$ is incomparable to all sets $F_{i}$ with $i>1$, hence $F_{i} \cap L_{t_{1}+1} \nsubseteq F_{1}$ for all $i>1$. In particular it is non-empty, hence all sets $F_{i}$, for $i=1, \ldots,(2 c)^{\ell}+1$, have an edge in $L_{t_{1}+1}$. The sets $F_{i}$ such that $t_{i}=t_{1}$ have an edge in $\mathcal{L}_{t_{1}}$ by definition. The sets $F_{i}$ such that $t_{i}>t_{1}$ have an edge in $\mathcal{L}_{t_{1}+1}$ as $\mathcal{L}_{t_{i}} \cap L_{t_{1}+1} \subseteq \mathcal{L}_{t_{1}+1}$, by definition of the sets $\mathcal{L}_{i}$. Since the size of $\mathcal{L}_{t_{1}} \cup \mathcal{L}_{t_{1}+1}$ is at most $2 c$, there exists a subset $B$ of $A$ of size at least $(2 c)^{\ell-1}+1$ of sets $F_{i}$ sharing a same edge $e \in \mathcal{L}_{t_{1}} \cup \mathcal{L}_{t_{1}+1}$. The set $\{F \backslash e \mid F \in B\}$ has size $|B| \geq(2 c)^{\ell-1}+1$ and is an antichain of sets of size at most $\ell-1$ by definition of $\preceq$. This contradicts the induction hypothesis.

By Dilworth's Theorem, there exists a partition of $\mathcal{L}$ into $(2 c)^{k}$ sets totally ordered by $\preceq$, which can be be refined according to the cardinality to obtain a partition into $k(2 c)^{k}$ correlated sets. Such a partition can be found in FPT time.

Let us now consider such a partition $\mathcal{F}_{1}, \mathcal{F}_{2}, \ldots, \mathcal{F}_{k(2 c)^{k}}$ of $\mathcal{L}$ into correlated sets. Observe that by Lemma 23 we can restrict our search to multicuts of the following type in $G_{1}$ :

- A backbone edge $v_{i} v_{i+1}$.

- Other edges in the lemon $M_{i}$, which separate $v_{i}$ from $v_{i+1}$ in $M_{i}$.

- Edges in $\mathcal{L}_{i}$.

- Edges in $\mathcal{R}_{i}$, which is defined analogously to $\mathcal{L}_{i}$, with the roles of vertices $x$ and $y$ reversed.

Due to space restrictions, the two following proofs are omitted. These proofs are quite technical and we refer the curious reader to a full version of the paper.

LEMMA 25. We can assume that there are no cherries $C_{i}$. Moreover, if a multicut of size at most $k$ exists, there exists one which contains only edges in one lemon $M_{i}$.

THEOREM 26. We can assume that $G_{1}$ only consists of the backbone $P_{1}$.

\subsection{Reducing to 2-SAT}

We are left with instances in which the $Y$-components with two attachment vertices consist of backbones. We now reduce the last components.

LEMma 27. We can assume that there is no component with one attachment vertex.

Proof. Let $Y=\left\{y_{1}, \ldots, y_{p}\right\}$ and $k$ be the number of free edges in our multicut. A vertex $y_{i} \in Y$ is safe if for each pair of components attached only to $y_{i}$ there are no internal requests. If $y_{i}$ is not safe then there is a request $\left(u, y_{i}, v\right)$ in the union of the two components attached to $y_{i}$, hence $y_{i}$ must be disconnected from $u$ or from $v$ by the solution. We explore one branch where $u$ is added to $Y$, and one branch where $v$ is added to $Y$. This creates a component with two 
attachment vertices. This component has a backbone, and then the number of free edges decreases.

Hence we can assume that all the vertices of $Y$ are safe. The $y_{i}$-cherry is the union of all the components attached to $y_{i}$. We branch over all possible integer partitions of $k$ into a sum $k_{1}+k_{2}+\cdots+k_{p}=k$. In each branch, we require that $k_{i}$ edges are deleted in the $y_{i}$-cherry for $i=1, \ldots, p$. By Lemma 10, the $y_{i}$-cherry has a bounded active set $\mathcal{L}_{i}$, hence in the $y_{i}$-cherry we can consider only a bounded number of cuts of size $k_{i}$ : all subsets of $\mathcal{L}_{i}$ of size $k_{i}$. We then branch over these different choices. In a given branch, we delete a particular set of edges $F_{i}$ in the $y_{i}$-cherry. Thus, we delete the vertices of the $y_{i}$-cherry isolated from $y_{i}$ by $F_{i}$, and contract the other vertices of the $y_{i}$-cherry to $y_{i}$. Finally, no $Y$-cherry remains.

THEOREM 28. Multicut is FPT.

Proof. By Lemma 27, we are only left with a graph $G$ which is a subdivision of a graph with at most $k$ edges, and a multicut must consist of exactly one edge in each subdivided edge. Let us consider a half-request $\left(v_{i}, x, v_{j}^{\prime}\right)$. Assume without loss of generality that $v_{i} \in G_{1}, v_{j}^{\prime} \in G_{2}$, and $x$ belongs to $G_{1}$ and $G_{2}$ (if $x$ does not belong to $G_{1}$ or $G_{2}$, then splitting $Y$ automatically results in cutting the half-request $\left.\left(v_{i}, x, v_{j}^{\prime}\right)\right)$. For simplicity, we assume that the edges of both $P_{1}$ and $P_{2}$ are enumerated in increasing order from $x$. We add to $\mathcal{C}$ the clauses $x_{1} \geq i \Rightarrow x_{2} \leq j-1$ and $x_{2} \geq j \Rightarrow x_{1} \leq i-1$. We transform all the half requests in this way. Hence we are only left with a set of clauses which we have to satisfy.

We add all the relations $x_{i} \geq a \Rightarrow x_{i} \geq a-1$ and $x_{i} \leq a \Rightarrow x_{i} \leq a+1$ and $x_{i} \geq a \Rightarrow \neg\left(x_{i} \leq a-1\right)$ and $x_{i} \leq a \Rightarrow \neg\left(x_{i} \geq a+1\right)$. We now have a 2 -SAT instance which is equivalent to the original multicut instance. As 2SAT is solvable in polynomial time, this shows that BACKBONE MULTICUT is FPT. Hence the simpler COMPONENT MULTICUT problem is FPT. Together with Theorem 12 which reduces MULTICUT to COMPONENT MULTICUT, this concludes the proof of Theorem 28 .

We do not detail the running time of the overall algorithm, but it is not hard to see that it is single exponential. The branching tree has a polynomial depth as the invariants are polynomially bounded, and the amount of work at each node and the branching degree are bounded by single exponentials in $k$.

We think that it should be possible to adapt our proof for edge-multicut in terms of vertex-multicut.

\section{REFERENCES}

[1] N. Bousquet, J. Daligault, S. Thomassé, and A. Yeo. A polynomial kernel for multicut in trees. In $S T A C S$, volume 09001 of Dagstuhl Seminar Proceedings, pages 183-194, 2009.

[2] S. Chawla, R. Krauthgamer, R. Kumar, Y. Rabani, and D. Sivakumar. On the hardness of approximating multicut and sparsest-cut. Comput. Complex., 15(2):94-114, 2006.

[3] J. Chen, Y. Liu, and S. Lu. An improved parameterized algorithm for the minimum node multiway cut problem. Algorithmica, 55(1):1-13, 2009.

[4] J. Chen, Y. Liu, S. Lu, B. O'Sullivan, and I. Razgon. A fixed-parameter algorithm for the directed feedback vertex set problem. In $S T O C$, pages 177-186, 2008.
[5] M.-C. Costa, L. Létocart, and F. Roupin. Minimal multicut and maximal integer multiflow: A survey. European Journal of Operational Research, 162(1):55-69, 2005.

[6] J. Daligault, C. Paul, A. Perez, and S. Thomassé. Reducing multicut to bounded tree-width. http://www.lirmm.fr/ daligault/Reduction.pdf, 2010.

[7] R. G. Downey and M. R. Fellows. Parameterized Complexity. Springer, 1999.

[8] J. Flum and M. Grohe. Parameterized complexity theory. Springer-Verlag New York Inc, 2006.

[9] N. Garg, V. Vazirani, and M. Yannakakis. Approximate max-flow min-(multi)cut theorems and their applications. In STOC, pages 698-707, 1993.

[10] N. Garg, V. Vazirani, and M. Yannakakis. Multiway cuts in directed and node weighted graphs. In Automata, Languages and Programming, volume 820 of Lecture Notes in Computer Science, pages 487-498, 1994.

[11] N. Garg, V. Vazirani, and M. Yannakakis. Primal-dual approximation algorithms for integral flow and multicut in trees. Algorithmica, 18(1):3-20, 1997.

[12] G. Gottlob and S. Tien Lee. A logical approach to multicut problems. Inf. Process. Lett, 103(4):136-141, 2007.

[13] S. Guillemot. FPT algorithms for path-transversals and cycle-transversals problems in graphs. In IWPEC, pages 129-140, 2008.

[14] J. Guo, F. Hüffner, E. Kenar, R. Niedermeier, and J. Uhlmann. Complexity and exact algorithms for multicut. In SOFSEM, pages 303-312, 2006.

[15] J. Guo and R. Niedermeier. Fixed-parameter tractability and data reduction for multicut in trees. Networks, 46(3):124-135, 2005.

[16] D. Marx. Parameterized graph separation problems. Theor. Comput. Sci., 351(3):394-406, 2006.

[17] D. Marx, B. O'Sullivan, and I. Razgon. Treewidth reduction for constrained separation and bipartization problems. In STACS, 2010.

[18] D. Marx and I. Razgon. Constant ratio fixed-parameter approximation of the edge multicut problem. In ESA, volume 5757 of Lecture Notes in Computer Science, pages 647-658. Springer, 2009.

[19] D. Marx and I. Razgon. Fixed-parameter tractability of multicut parameterized by the size of the cutset. In STOC, 2011.

[20] H. Nagamochi and T. Ibaraki. Algorithmic Aspects of Graph Connectivity. Cambridge University Press, New York, NY, USA, 1 edition, 2008.

[21] R. Niedermeier. Invitation to Fixed Parameter Algorithms (Oxford Lecture Series in Mathematics and Its Applications). Oxford University Press, USA, March 2006.

[22] N. Robertson and P.D. Seymour. Graph minors. XIII. The disjoint paths problem. J. Combin. Theory, Ser. $B, 63(1): 65-110,1995$. 